\title{
SHEAR LAYER INSTABILITIES IN A COUNTER-FLOWING WALL JET
}

\author{
Sachin Sharma \\ Mechanical, Automotive \& Materials Engineering \\ University of Windsor \\ Windsor, Canada \\ sharm14d@uwindsor.ca
}

\author{
Ram Balachandar \\ Civil and Environmental Engineering \\ University of Windsor \\ Windsor, Canada \\ rambala@uwindsor.ca
}

\author{
Vimaldoss Jesudhas \\ Civil and Environmental Engineering \\ University of Windsor \\ Windsor, Canada \\ jesudha@uwindsor.ca \\ Ron Barron \\ Mathematics and Statistics \\ University of Windsor \\ Windsor, Canada \\ az3@uwindsor.ca
}

\begin{abstract}
Counter-flowing wall jets are often used in heat transfer and combustion applications. While several experimental and numerical studies have been conducted to evaluate the characteristics of counter-flowing wall jets, their internal turbulence structure is not well understood. This paper presents the results of a three-dimensional, unsteady, Improved Delayed Detached Eddy Simulation conducted on a counter-flowing wall jet. The mean flow features captured by the simulation are presented with relevant discussions. The shear layer instabilities caused by the vortical structures in the recirculation zone are analyzed by examining the instantaneous flow field. The flow mechanism that causes the fluctuations in the penetration length is also evaluated. The results depict the complexity of the turbulence structure in a counter-flowing wall jet.
\end{abstract}

Keywords- Counter-flowing jets; turbulent wall jet; IDDES; shear layer instability; penetration length fluctuations

\section{INTRODUCTION}

A turbulent wall jet issued opposite to the direction of a moving main / ambient stream is known as a counter-flowing wall jet (CFWJ). Counter-flowing wall jets are particularly effective as mixing devices, such as effluent mixing in rivers and streams, air-fuel mixture for combustion, etc. This is due to the fact that the turbulence in a counter-flowing wall jet is more enhanced than cross and co-flowing jets or even a counter-flowing free jet (CFFJ). Fig. 1a shows the schematic of a typical CFWJ flow field. The higher momentum at the inlet enables the CFWJ to penetrate the main flow. The momentum of the CFWJ reduces gradually as it approaches the stagnation point. The main flow pushes the jet backwards, thereby creating a recirculation zone. The recirculation zone enhances the turbulence in the CFWJ, thereby improving its mixing efficiency. The distance from the nozzle exit to the stagnation point is known as the penetration length of the jet $\left(x_{s}\right)$. The dark black line in Fig. 1a shows a streamsurface, which is the loci of $U=U_{o}$. This streamsurface divides the wall jet and main flow region. The maximum height of this streamsurface gives the width $(h)$ of the recirculation zone. The $U=0$ line divides the forward and backward flow. While several experimental studies [1-4] have been conducted on CFFJ, the studies on CFWJ are relatively limited. However, the studies of CFFJ can be used to interpret the salient characteristics of a CFWJ flow field. One of the prominent aspects of the CFFJ is the fluctuating nature of the penetration length $[2,4]$. The stagnation point oscillates about a mean position. The presence of the wall in a CFWJ further enhances the complexity of the flow. In experimental studies, it is difficult to capture the penetration length accurately. Typically, experimental researchers have employed dye or wool turfs to identify the instantaneous location of the stagnation point $[1,5]$, leading to uncertainties in the measurements. Also, in experiments the complete threedimensional (3D) flow field is not available for analysis, thereby posing challenges in identifying the flow physics that are responsible for the oscillations of the penetration length.

Numerical studies of CFWJ are sparse. "Reference [6]" studied the mean characteristics of CFWJ using the ReynoldsAveraged Navier-Stokes (RANS) $k-\varepsilon$ turbulence model. They analyzed the mean characteristics of the flow for different velocity ratios $\alpha=U_{j} / U_{o}$ where $\alpha$ is the ratio of jet velocity $\left(U_{j}\right)$ to main flow velocity $\left(U_{o}\right)$.However, due to the limitations of RANS turbulence models, a rigorous investigation of the turbulence characteristics was not carried out. To address these limitations, 3D, unsteady, Improved Delayed Detached Eddy Simulation (IDDES) of a planar CFWJ is conducted in this study. After validating the simulation results with available experimental data, the instantaneous flow field is analyzed. The shear layer instabilities in the CFWJ flow field are identified. The flow 
mechanism responsible for the penetration length fluctuations is also identified.

\section{MODEL SETUP}

In the present study, a hybrid RANS-LES approach was employed to model turbulence. IDDES uses RANS near the walls and LES away from it. The complete formulation is available in several publications [7,8] and hence not repeated here. The commercial CFD solver STAR-CCM+ was used to setup and run the simulations. A two-dimensional representation of the $3 \mathrm{D}$ computational domain is shown in Fig. 1b. The computational domain is modeled based on the experiments of [9]. A hexahedral mesh was used, with refinements in the regions of interest. Based on the grid dependency study, the mesh selected for the present simulation consists of about 10 million cells. .Fine prism layers were added near the walls. The boundary conditions used in the simulation are also shown in Fig. 1b. The side walls were treated as no-slip walls; the complete details of the simulated case are presented in Fig. 1b. Shear stress transport (SST) $k-\omega$ is used along with LES in the present simulation. IDDES uses a blending function to switch between the RANS and LES regions; this was monitored to ensure that LES was executed in the regions of interest. The solution was considered to be converged when the residuals for continuity and momentum fell below $10^{-6}$. The mean quantities presented here were obtained by averaging for a time of 50s following convergence
The simulation results were validated based on the experiments of [9]. The validation procedure was based on the American institute of Aeronautics and Astronautics (AIAA) guidelines for verification and validation of CFD simulations (2002). The penetration length, width of the recirculation zone and velocity profiles were compared with the experimental results. The simulation results agreed well with the experimental results. Only after establishing sufficient confidence in the simulation results, the analysis presented here was carried out.

\section{RESULTS AND DISCUSSION}

Fig. 2 shows the velocity vectors in the mean flow field of the CFWJ predicted by the simulation. The velocity vectors are colored based on the mean streamwise velocity. As the jet exists the nozzle at $\left(x / h_{j}=0\right)$, where $h_{j}$ is the size of jet, a shear layer is created between the jet and recirculation zone. The mean velocity of the jet decreasing as it approaches the stagnation point. At the stagnation point $\left(x / h_{j}=72\right)$ the jet turns in the opposite direction due to the influence of the main flow, forming the recirculation zone. The $U=0$ line divides the forward and backward momentum in the flow field. Due to the interaction between the two flows, the main flow readjusts by flowing around the recirculation zone. Figs. 3(a-d) depict Instantaneous velocity at four time instances. As the jet emanates from the nozzle, the vortical structures from recirculation region interact with the shear layer and create

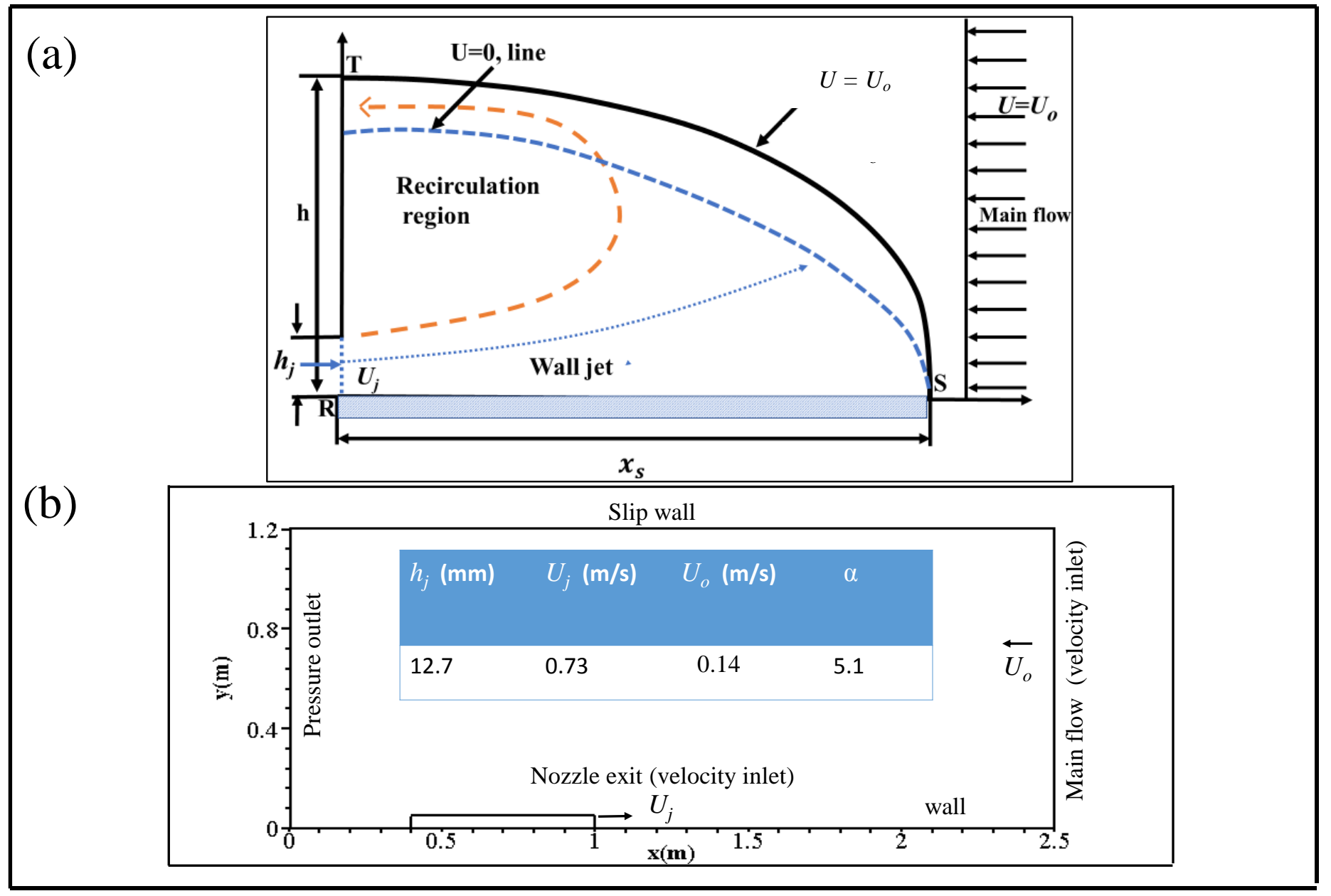

Fig. 1(a) Schematic of counter-flowing wall jet (b) Computational domain 
instabilities. The vectors are also superimposed to depict this interaction. The downward motion of the fluid in the recirculation zone (marked by red arrow in Fig. 3a) interacts with the jet between $x / h_{j}=20$ to 30. This excites the jet causing instabilities making it wavy in nature. The influence of the recirculation zone and the adverse pressure gradient it encounters enables it to detach from the wall. This allows the main flow to penetrate further into the jet in the near bed region (marked by the dashed circle in Fig. 3a). As the fast moving jet negotiates the slow moving fluid particles (light blue region) arriving from the near-wall main flow, it bifurcates in to an upward moving flow towards the $U=0$ line and the wall (Fig. 3b). The fluctuating nature of the wall jet continues (Fig. 3c and Fig. 3d) and the corresponding main flow adjusts itself to the jet characteristics, reducing the penetration length of the jet. The penetration length is $x / h_{j}=$ 55 in Fig. 3d. The phenomenon of jet fluctuations and the corresponding change in the penetration length enhances the mixing. This analysis clearly shows the complex nature of the turbulence in the CFWJ flow field and the feedback

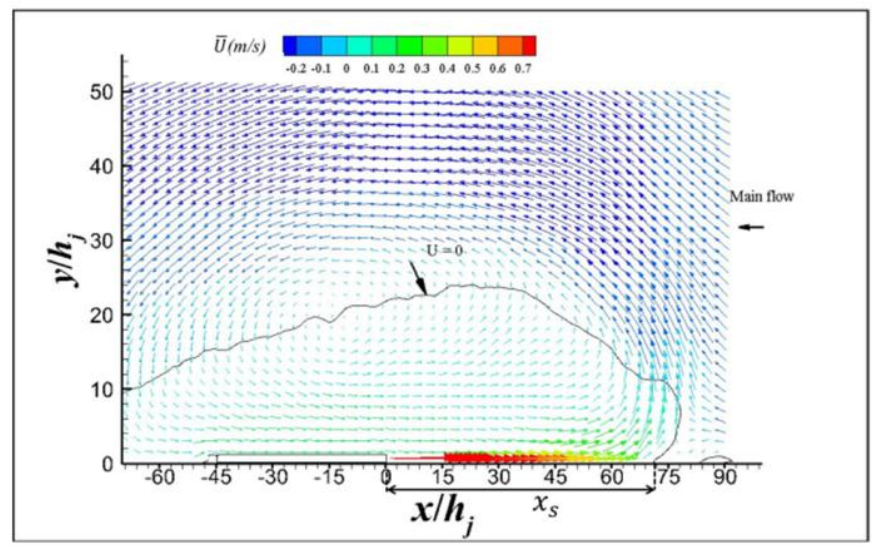

Fig. 2 Mean flow field of counter-flowing wall jet mechanism that exists between the recirculation zone and the wall jet. Further analysis is needed to explore this phenomena.

\section{CONCLUSION}

A computational study of a counter-flowing wall jet is carried out using the 3D, unsteady, Improved Delayed Detached Eddy Simulation. The simulation captures the relevant flow features of the CFWJ flow field. The mechanism causing the fluctuations in the penetration length has been identified. The analysis of the instantaneous velocity field shows a feedback mechanism that exists between the recirculation zone and the jet, causing the oscillation of the jet penetration length. This mechanism contributes to the enhanced mixing in the CFWJ flow field.

\section{REFERENCES}

[1] W. D. Morgan, B. J. Brinkworth, and G. V. Evans, "Upstream penetration of an enclosed counterflowing jet," Industrial \& Engineering Chemistry Fundamentals, vol. 15, no. 2, pp. 125-127, (1976).

[2] M. Yoda and H. E. Fiedler, "The round jet in a uniform counterflow: flow visualization and mean concentration measurements," Experiments in Fluids, vol. 21, no. 6, pp. 427-436, (1996)

[3] K. M. Lam and H. C. Chan, "Round jet in ambient counterflowing stream," Journal of Hydraulic Engineering, vol. 123, no. 10, pp. 895903, (1997).

[4] S. Bernero and H. E. Fiedler, "Application of particle image velocimetry and proper orthogonal decomposition to the study of a jet in a counterflow," Experiments in Fluids, vol. 29, no. 1, pp. S274-S281, 2000

[5] R. Balachandar, L. Robillard, and A. S. Ramamurthy, "Some characteristics of counter flowing wall jets," Journal of Fluids Engineering, vol. 114, no. 4, pp. 554-558, (1992).

[6] H. Kordi, A. Abedini, and E. Kordi, "Numerical modeling of the penetration area of reversed submerged jet by the methods of finite elements and finite volumes," Water Resources, vol. 43, no. 3, pp. 472477, (2016).

[7] M. L. Shur, P. R. Spalart, M. K. Strelets, and A. K. Travin, "A hybrid RANS-LES approach with delayed-DES and wall-modelled LES capabilities," International Journal of Heat and Fluid Flow, vol. 29, no. 6, pp. 1638-1649, (2008).

[8] V. Jesudhas, Modelling of Free-surface Flows with Air Entrainment, Ph.D. Dissertation, Univ. of Windsor, Windsor, ON, Canada, (2016).

[9] Tudor, M., Turbulent Characterstics of a counter flowing wall jets, Master's Thesis, University of Concordia, (2003).

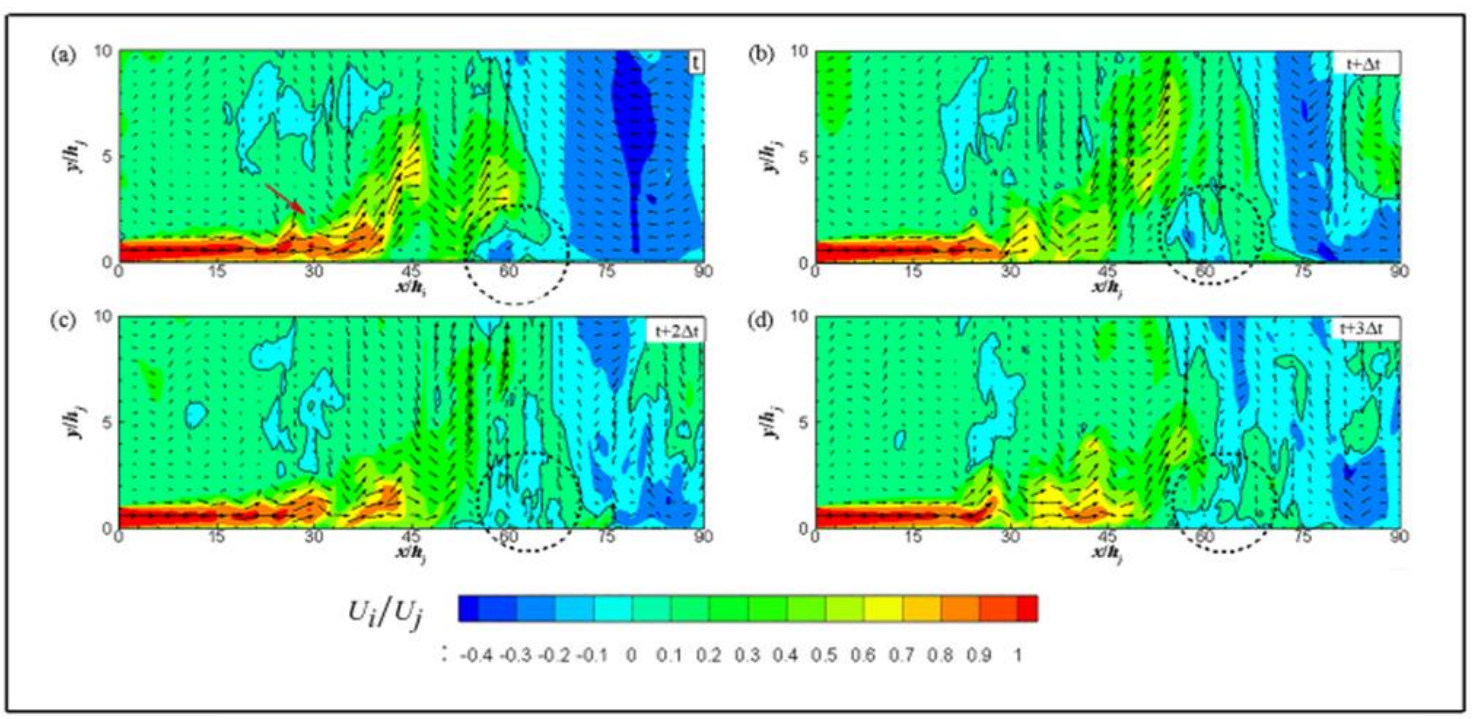

Fig. 3 Instantaneous streamwise velocity at (a) $120 \mathrm{~s}$, (b) $120.5 \mathrm{~s}$, (c) $121 \mathrm{~s}$, (d) $121.5 \mathrm{~s}$ 